\title{
Risk factors of delayed graft function in deceased donor kidney transplantation
}

\author{
Seung Hwan Song ${ }^{1}$, Dami Jung ${ }^{2}$, Ku Yong Chung ${ }^{2}$ \\ ${ }^{1}$ Division of Transplantation Surgery, Department of Surgery, Ewha Womans University Seoul Hospital, Seoul, Korea \\ ${ }^{2}$ Division of Transplantation Surgery, Department of Surgery-Transplantation, Ewha Womans University Mokdong Hospital, Seoul, Korea
}

Background: The most significant complication is delayed graft function (DGF) on deceased donor kidney transplantation (DDKT). Multiple factors belonging to donor, recipient, and transplant procedures have an effect on the development of DGF. We aim to evaluate the predictable risk factors of DGF and its effects on the graft function and survival in our country.

Methods: Between January 2008 and October 2020, a total of 99 recipients who underwent DDKT were retrospectively reviewed. We classified recipients into two groups; DGF(-) vs. DGF(+). The risk factors of DGF associated with donor and recipient were analyzed. The effects of DGF were examined on the graft function and survival.

Results: We included 99 DDKT cases. Among 99 DDKT, 35 cases were included in DGF $(+)$, and the others were in DGF $(-)(n=64)$. A cold ischemic time (CIT) of the DGF(+) group was $297.4 \pm 96.2$ minutes, compared to $248.2 \pm 60.7$ minutes of the DGF $(-)$ group $(P=0.002)$. Shorter dialysis duration and marginal donors were observed to be statistically significant compared to recipients without DGF $(P=0.05)$. The serum creatinine level before donor nephrectomy of the $D G F(+)$ group was significantly higher $(1.7 \pm 1.1$ $\mathrm{mg} / \mathrm{dL})$ than that of the $\mathrm{DGF}(-)$ group $(1.0 \pm 0.5 \mathrm{mg} / \mathrm{dL}, P<0.0001)$. There was no significant difference in graft function and survival between $\mathrm{DGF}(+)$ and $\mathrm{DGF}(-)$ group.

Conclusions: Longer CIT, marginal donor, and the serum creatinine level before donor nephrectomy are the most important risk factors for DGF. There was no significant difference in graft function and survival between DGF(+) and DGF(-) group.

Corresponding author: Seung Hwan Song

E-mail: seunghwanhappy@ewha.ac.kr

(C) The Korean Society for Transplantation

This is an Open Access article distributed under the terms of the Creative Commons Attribution Non-Commercial License (http://creativecommons.org/licenses/by-nc/4.0/) which permits unrestricted non-commercial use, distribution, and reproduction in any medium, provided the original work is properly cited. 